\title{
早更新世赤道太平洋上部水体结构的东西向不对称 格局的形成
}

\author{
金海燕，翦知淐，成金荣，郭建卿 \\ 同济大学海洋地质国家重点实验室, 上海 200092 \\ E-mail: jinhy@tongji.edu.cn
}

2010-12-17 收稿, 2011-04-29 接受

国家自然科学基金(40806020)、国家重点基础研究发展计划(2007CB815901)、高等学校博士学科点基金(200802471034)和大洋协会“十一五” 课题(DY115-01-2-3)资助

\begin{abstract}
摘要 选取大洋钻探 ODP 130 航次在西太平洋暖池钻取的 807 站 A 孔上段 $43 \mathrm{~m}$ 岩芯样 品为研究材料, 进行浮游有孔虫表层水种、次表层水种的氧、碳稳定同位素分析, 并与赤 道东太平洋 ODP 851 站的记录比较, 揭示了赤道太平洋海区距今 $2.5 \mathrm{Ma}$ 以来上部水体的 古海洋变迁. 研究发现, 早更新世 1.6 1.4 Ma 时, 赤道东、西太平洋表层水和次表层水的 氧同位素都发生明显的分异, 进一步证实赤道太平洋类似于现代的东-西向温度梯度(表 层水温西高东低)和上部水体温跃层西深东浅的不对称格局在当时最终形成. 由于次表 层水的氧同位素东-西向梯度变化大于表层水的, 说明赤道东、西太平洋这种不对称格局 的形成应该与东太平洋温跃层变浅、次表层水显著降温关系更为密切. 此外, $1.6 \mathrm{Ma}$ 之 后, 赤道太平洋表层水和次表层水的碳同位素差异明显变小, 且其偏心率长周期也从 $400 \mathrm{ka}$ 变为 $500 \mathrm{ka}$, 反映了大洋碳储库的改组, 可能与这一时期南大洋深部水的改组及 其对热带太平洋(特别是次表层海水)的影响加剧有关, 说明热带系统可以在全球气候变 化中起重要作用.
\end{abstract}

\section{关键词}

赤道太平洋

氧、碳同位素

东、西向不对称格局

次表层水

早更新世
近年来随着对厄尔尼诺和季风气候的注意, 低 纬热带海区在全球气候系统中的关键作用已经得到 广泛的认可. 特别是在赤道太平洋海区, 其西边有西 太平洋暖池 ${ }^{[1]}$, 东边有 “冷舌”, 因而形成了长期、且 相对稳定的赤道太平洋表层水温西高东低的东-西向 温度梯度及上部水体温跃层西深东浅的不对称格局. 一旦这种格局发生变化, 低纬的气候信号就会通过 水气交换传输到中高纬海区, 进而影响到全球的气 候系统 ${ }^{[2,3]}$. 因此, 赤道太平洋这种东-西向温度梯度 和上部水体不对称格局的形成时间和原因自然成为 热带太平洋古海洋与古气候学研究的热点. 最近, 美 国 Wara 等人 ${ }^{[4]}$ 利用表层浮游有孔虫的 $\mathrm{Mg} / \mathrm{Ca}$ 比值恢 复赤道太平洋海区的古温度, 发现赤道太平洋的东-
西向温度梯度在距今 1.6 Ma 最终形成 ${ }^{[4 \sim 6]}$. 进一步 的研究表明, 早更新世 1.6 Ma 时, 南大洋中层水和 深层水的化学分隔(chemical divide)形成 ${ }^{[7]}$, 这种高 纬海区大洋环流的重组, 不仅可能导致热带太平洋 上部水体垂向结构发生变化、出现东西向不对称的格 局 ${ }^{[8]}$, 而且可以引起大洋碳储库的变动, 譬如南沙海 区 ODP 1143 站碳同位素的偏心率长周期“节律失调”、 由 $400 \mathrm{ka}$ 变为 $500 \mathrm{ka}^{[9]}$. 然而, 以往关于早更新世热 带太平洋东、西向不对称性的研究主要侧重于表层海 水温度 ${ }^{[4-8]}$, 而忽略了热带次表层海水的变化. 实际 上, 现代海洋学研究表明, 热带太平洋次表层水的温 度异常与 ENSO 的发生有着更为直接的关系 ${ }^{[10,11]}$, 且 有迹象表明在晚第四纪冰期旋回中热带次表层海水

英文版见: Jin H Y, Jian Z M, Cheng X R, et al. Early Pleistocene formation of the asymmetric east-west pattern of upper water structure in the equatorial Pacific ocean. Chinese Sci Bull, 2011, 56, doi: 10.1007/s11434-011-4547-3 
的变化比表层水的更为显著 ${ }^{[12,13]}$, 说明热带次表层 海水在气候演变中的重要性和特殊性. 为此, 本次工 作以西太平洋暖池核心区的 ODP 807 站岩芯为研究 材料, 旨在通过浮游有孔虫表层水种、次表层水种的 氧、碳稳定同位素分析, 并与赤道东太平洋 ODP 851 站的相关记录 ${ }^{[14]}$ 比较, 揭示晚上新世 $2.5 \mathrm{Ma}$ 以来热 带太平洋海区上部水体的演变历史, 探讨次表层海 水在早更新世热带太平洋东、西向不对称格局形成过 程中的变化, 为研究赤道太平洋海区在气候演变中 的作用提供新的科学依据.

\section{1 研究材料与方法}

本次工作采用的材料来自于大洋钻探 (ODP)第 130 航次在赤道西太平洋翁通-爪哇海台钻取的 807 站岩芯 $\left(3^{\circ} 36.42^{\prime} \mathrm{N}, 156^{\circ} 37.49^{\prime} \mathrm{E}\right.$, 水深 $2803.8 \mathrm{~m}$, 图 1), 其中 A 孔长 $822.9 \mathrm{~m}$, 取其上部 $43 \mathrm{~m}$ 的岩芯进行分析 工作. 该段沉积物的岩性均一, 主要由浅灰色-白色 的微体化石软泥构成, 伴有生物扰动现象. 根据大洋 钻探航次报告 ${ }^{[15]}$, 807 站 $\mathrm{A} 孔 43 \mathrm{~m}$ 岩芯底部的年龄 约为第四纪 $2.5 \mathrm{Ma}$ 左右, 研究工作按 5 10 $\mathrm{cm}$ 不等 间隔取样, 共获得样品 860 个. 对所有样品中浮游有 孔虫表层水种 Globigerinoides ruber 和次表层水种 Pulleniatina obliquiloculata 进行稳定氧、碳同位素测 试, 测试精度参照中国国家碳酸钻标准(GBW04405) 和国际标准(NBS19), 2005 年 $\delta^{18} \mathrm{O}$ 和 $\delta^{13} \mathrm{C}$ 的标准误差 分别是 $0.08 \%$ 和 $0.06 \%$ (PDB, Pee Dee Belemnite). 浮

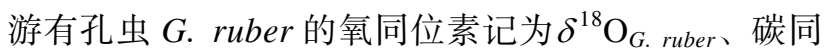
位素记为 $\delta^{13} \mathrm{C}_{\text {G. ruber }}$; P. obliquiloculata 的氧同位素记 为 $\delta^{18} \mathrm{O}_{P}$. obliquiloculata、碳同位素记为 $\delta^{13} \mathrm{C}_{P}$. obliquiloculata. 氧、碳稳定同位素的测试在同济大学海洋地质国家重 点实验室完成.

\section{2 地层年代框架}

在原有的生物地层学和磁性地层学事件 ${ }^{[15]}$ 基础 上, 增加了浮游有孔虫 G. ruber 粉红色壳体的末次出 现面(LAD) $1.92 \mathrm{~m}$ (距今 12 万年前)和初次出现面 (FAD)6.67 m(距今约 40 万年前)这 2 个时间控制点, 再对比 LR04 全球底栖有孔虫氧同位素曲线 ${ }^{[16]}$, 建立 起 ODP 807 站 A 孔的年代地层剖面(图 2). 从图 2 中 可以看出, 该站位上部 $43 \mathrm{~m}$ 岩芯记录了早第四纪 2.5 $\mathrm{Ma}$ 以来的沉积历史, 共包含了深海氧同位素期 (MIS)1 99 个期次, 样品的平均时间分辨率为 $3 \mathrm{ka}$.

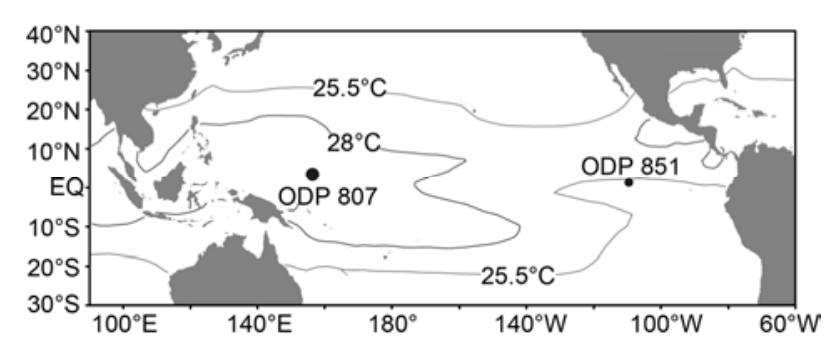

图 1 研究站位图

$28^{\circ} \mathrm{C}$ 等温线标示西太平洋暖池边界, ODP 851 为东太平洋站
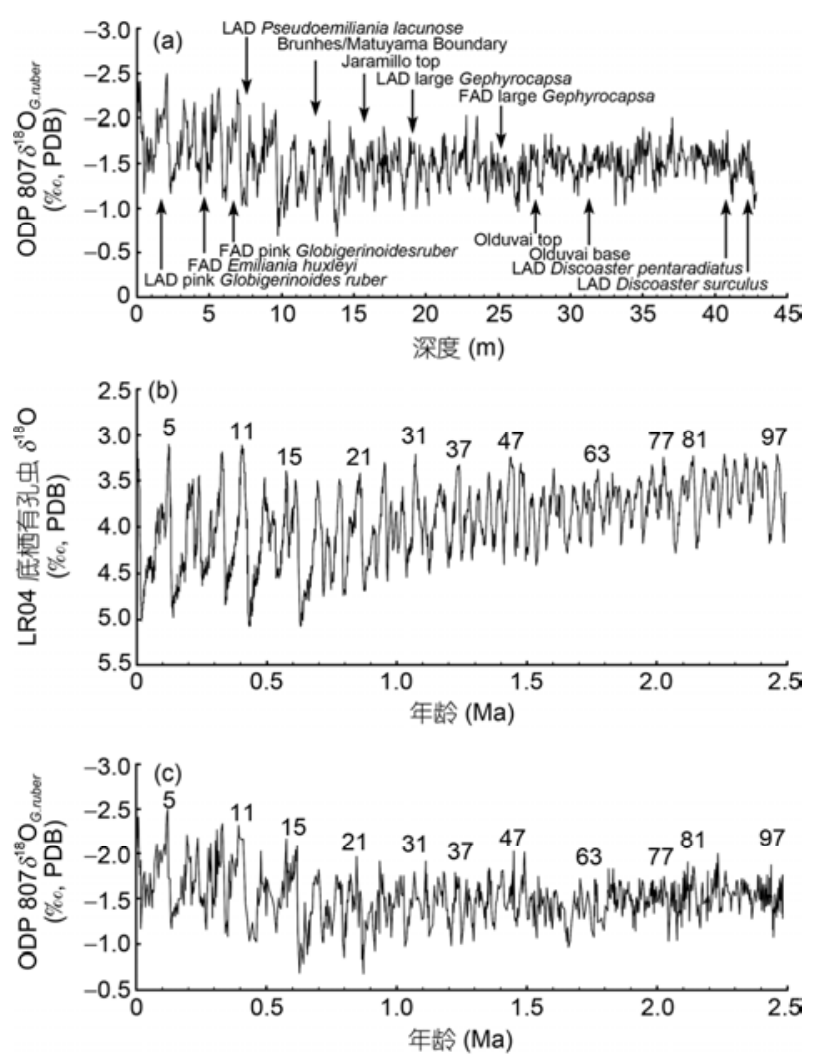

图 $20 D P 807$ 站年代地层剖面

(a) ODP 807 站深度-氧同位素曲线; (b) LR04 全球底栖有孔虫氧同位 素曲线 ${ }^{[16]}$; (c) ODP 807 站年龄-氧同位素曲线. (a)中箭头指示生物地层 和磁性地层年龄控制点所在深度, (b) 和(c)中曲线上方数字标示氧同位 素期次

\section{3 赤道太平洋表层水和次表层水氧同位素 的东-西向梯度变化}

赤道西太平洋 ODP 807 站浮游有孔虫氧同位

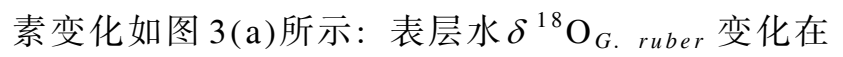
$-0.68 \%$ - $2.50 \%$ 之间, 平均值为 $-1.52 \%$; 次表层水 $\delta^{18} \mathrm{O}_{P .}$ obliquiloculata 变化在 $0.15 \%$ - $1.85 \%$ 之间, 平均值 为 $-0.96 \%$ 。 根据浮游有孔虫生态资料 ${ }^{[17]}$, 表层水种 
(a)

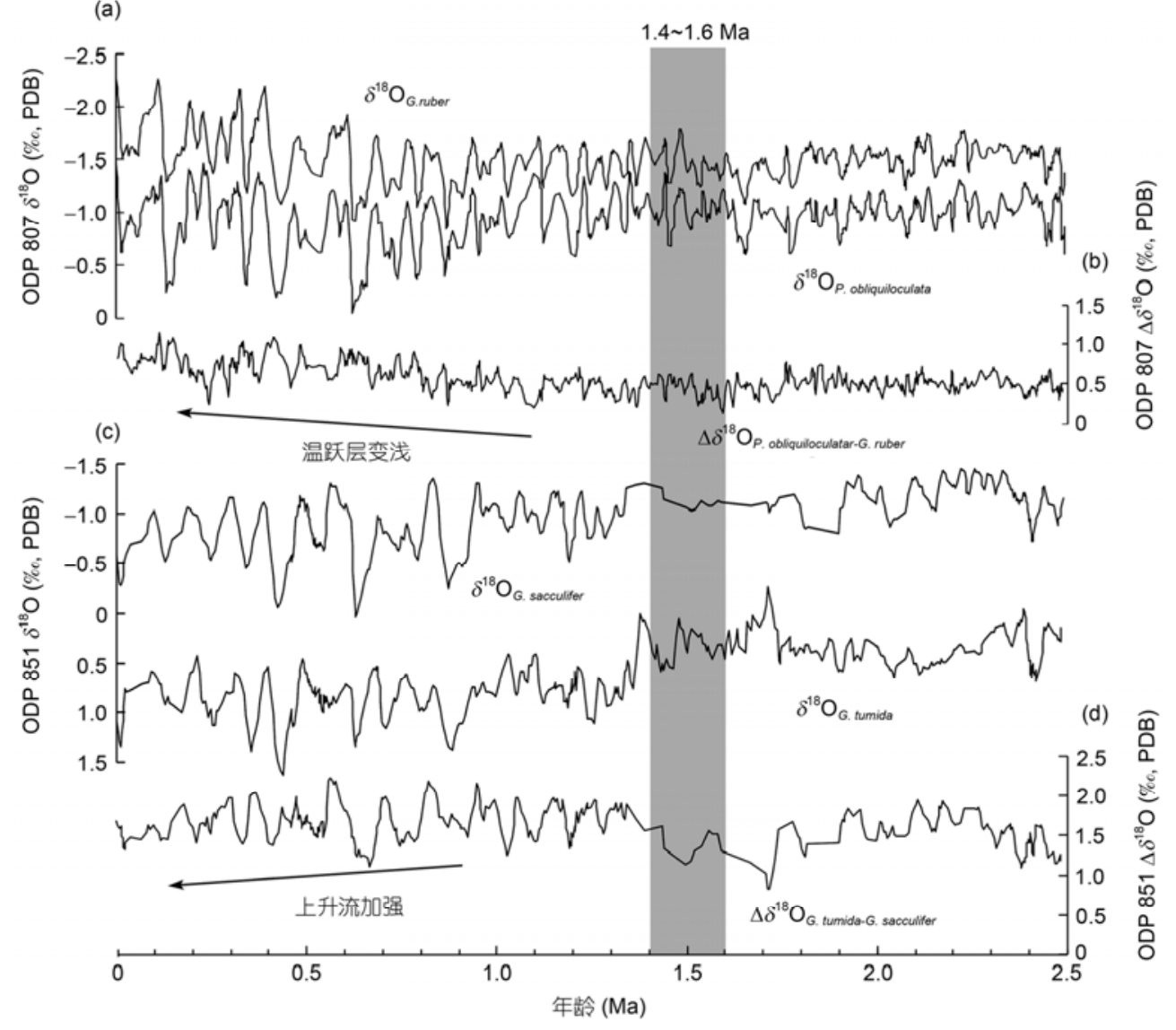

图 3 赤道太平洋 $2.5 \mathrm{Ma}$ 年以来的氧同位素变化

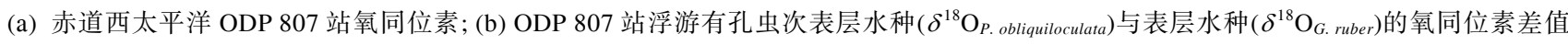
$\left(\Delta \delta^{18} \mathrm{O}_{\text {P. obliquiloculata-G. rubber }}\right.$; (c) 赤道东太平洋 ODP 851 站氧同位素; (d) ODP 851 站浮游有孔虫次表层水种 $\left(\delta^{18} \mathrm{O}_{G . ~ t u m i d a}\right)$ 与表层水种 $\left(\delta^{18} \mathrm{O}_{\text {G. sacculifer }}\right)$

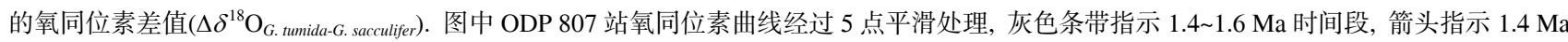
后东、西太平洋次表层水与表层水氧同位素差值的变化趋势

G. ruber 生活在水深 $50 \mathrm{~m}$ 以浅的海水上层, 而次表层 水种 P. obliquiloculata 生活在上部温跃层 ${ }^{[18]}$ 、水深 100

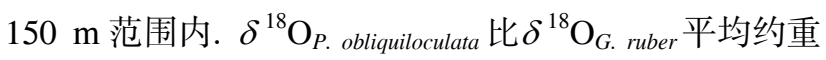
$0.56 \%$, 按照温度每下降 $1^{\circ} \mathrm{C}$ 氧同位素增重 $0.26 \%$ 来估 算 ${ }^{[19]}, \delta^{18} \mathrm{O}_{P \text {. obliquiloculata }}$ 代表的次表层水温度比 $\delta^{18} \mathrm{O}_{G \text {. ruber }}$ 代表的表层水温度约低 $2^{\circ} \mathrm{C}$ 左右. 现代海洋实际观测 资料表明(据 WOA 05 数据 ${ }^{[20]}$ ), 西太平洋翁通-爪哇 海台水深 $50 \mathrm{~m}$ 处的海水温度在 $28.5 \sim 29.0^{\circ} \mathrm{C}$ 之间, 水 深 $100 \mathrm{~m}$ 处的海水温度在 $26.5 \sim 27.0^{\circ} \mathrm{C}$ 之间, 温差为 $2{ }^{\circ} \mathrm{C}$, 说明氧同位素计算结果与实际资料相符. 因而, 可以用 G. ruber 和 P. obliquiloculata 的同位素分别代 表赤道西太平洋表层水和次表层水的同位素变化.

第四纪 $2.5 \mathrm{Ma}$ 以来, 赤道西太平洋 ODP 807 站 的表层水和次表层水氧同位素的变化趋势大致相同:
在距今 $0.6 \sim 1.2 \mathrm{Ma}$ 的“中更新世气候转型”事件 ${ }^{[21]}$ 之 前, 氧同位素变化幅度相对较小, 以 $41 \mathrm{ka}$ 斜率周期 为主; 而其后, 氧同位素变化幅度增大, 以 $100 \mathrm{ka}$ 偏 心率周期为主; 其间则为过渡期. 然而, 研究表明 “中更新世气候转型”可能始于 1.5 $\mathrm{Ma}$ 前 ${ }^{[22]}$, 与同一 时期赤道太平洋表层海水温度的东-西向不对称格局 最终形成相应 ${ }^{[4]}$. 如果计算 ODP 807 站次表层水和表

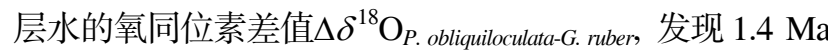
以来有变大的趋势(图 3(b)), 与东太平洋 ODP 851 站 $\left(2^{\circ} 46^{\prime} \mathrm{N}, 110^{\circ} 34^{\prime} \mathrm{W}\right.$, 水深 $\left.3760 \mathrm{~m}\right)$ 的次表层水和表层 水氧同位素差值 $\Delta \delta^{18} \mathrm{O}_{\text {G. tumida-G. sacculifer }}$ (采用浮游有孔 虫表层水种 Globigerinoides sacculifer 和次表层水种 Globorotalia tumida 的同位素，分别代表表层水和次 表层水的同位素变化 ${ }^{[14]}$, 并计算其差值)趋势不一样 
(图 3(d)). 在西太平洋, 次表层水和表层水的氧同位 素差值常用来指示海水垂向结构温跃层深度的变 化 ${ }^{[23,24]}$ : 如果表层混合作用增大、温跃层变深, 该差值 减小, 反之亦然. 然而在赤道东太平洋的上升流海区, 如果上升流增强、温跃层变浅, 导致次表层和表层水的 温度梯度减小, 因而次表层水和表层水的氧同位素差 值反而减小 ${ }^{[14]}$, 与西太平洋的情况正好相反. 因此, 距 今 $1.4 \mathrm{Ma}$ 以来, 赤道东、西太平洋的温跃层都有变浅 的趋势, 但两者的变化程度不一样: 在东太平洋, 温跃 层最显著的变浅发生在 1.7 1.4 Ma 期间(图 3(d)).

在赤道太平洋海区, 表层海水温度和氧同位素记 录都反映出1.6 1.4 Ma 之后西太平洋暖池表层水温略 有变暖, 而东太平洋“冷舌”持续变冷, 赤道太平洋上新 世 $5 \mathrm{Ma}$ 以来长期的 El Niño 状态结束 ${ }^{[4 \sim 6]}$, 东、西赤道 太平洋的不对称格局最终形成. 这一现象可以从赤道 东、西太平洋 ODP 851 站与 ODP 807 站的表层水氧同 位素差值 $\Delta \delta^{18} \mathrm{O}_{\text {G. sacculifer-G. ruber }}$ 的变化上得到印证. 图 4 揭示, 在 $2.5 \mathrm{Ma}$ 以来, 赤道东、西太平洋的次表层水氧 同位素差值 $\Delta \delta^{18} \mathrm{O}_{\text {G. tumida-P. obliquiloculata }}$ 明显大于表层水的

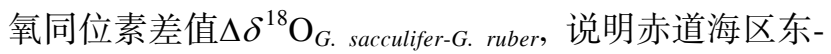
西向温度的不对称现象早已存在, 且主要表现在次表 层水上. 但是, 在 $1.4 \mathrm{Ma}$ 之后, $\Delta \delta^{18} \mathrm{O}_{\text {G. tumida-P. obliquiloculata }}$

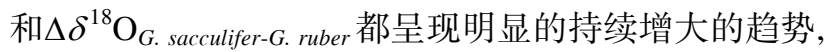
说明赤道太平洋东-西向温度的长期且相对稳定的不 对称格局最终形成. 选择 $0.6 \mathrm{Ma}$ (中更新世气候转型 期之后，开始出现 10 万年周期为主的时间)、1.4 1.6 $\mathrm{Ma}$ 为界(本次工作中赤道东、西太平洋不对称格局最
终形成的时间), 分别计算0 0.6, 0.6 1.4和1.6 2.5 Ma 这3个时间段内的平均值, 结果发现 ODP 851站与 ODP 807 站的次表层水氧同位素差值 $\Delta \delta^{18} \mathrm{O}_{\text {G. tumida-P. obliquiloculata }}$ (分别为 $1.75 \%$ o $1.69 \%$ 和 $1.40 \%$ ) 的变化都大于表 层水 $\Delta \delta^{18} \mathrm{O}_{\text {G. sacculifer-G. ruber }}$ 的 (分别为 $0.85 \%, 0.56 \%$ 。 和 $0.34 \%$ ). 特别是, 在距今 $1.4 \mathrm{Ma}$ 时, 次表层水 $\Delta \delta^{18} \mathrm{O}_{\text {G. tumida-P. obliquiloculata }}$ 突然增大近 $1.0 \%$ o(相当于赤道 太平洋次表层水的东-西向温度梯度增大 $4^{\circ} \mathrm{C}$ ), 明显 大于表层水 $\Delta \delta^{18} \mathrm{O}_{G \text {. sacculifer-G. ruber }}$ 的变化( 0.5\% ; 图4), 说明赤道东、西太平洋次表层水温度的不对称格局也 在早更新世1.6 1.4 Ma 期间形成, 且其变化较表层水 的更为显著.

\section{4 赤道东、西太平洋温度不对称格局的形 成原因探讨}

由于赤道东太平洋 ODP 851 站在 1.8 1.4 Ma 期 间的样品时间分辨率较低(图 3(c)), 本次研究结合前 人的研究结果 ${ }^{[4 \sim 6]}$, 将更新世 1.6 1.4 Ma 时间段作为 赤道太平洋东-西向温度的不对称格局最终形成的时 期. 这不仅体现在表层水的变化之上, 也反映在次表 层水的东-西向温度梯度突然显著增大之上, 并且次 表层水的变化更为明显. 正是由于这一时期赤道东 太平洋的温跃层变浅、上升流作用增强, 使得赤道东 太平洋次表层水显著降温( 1.4 Ma 前后, 西太平洋 $\delta^{18} \mathrm{O}_{P \text {. obliquiloculata }}$ 变化不大, 而东太平洋 $\delta^{18} \mathrm{O}_{G \text {. tumida 显 }}$ 著变重; 图 3(a)和(c)), 从而出现赤道太平洋海区温跃 层西深东浅的上部水体不对称格局. 可见, 早更新

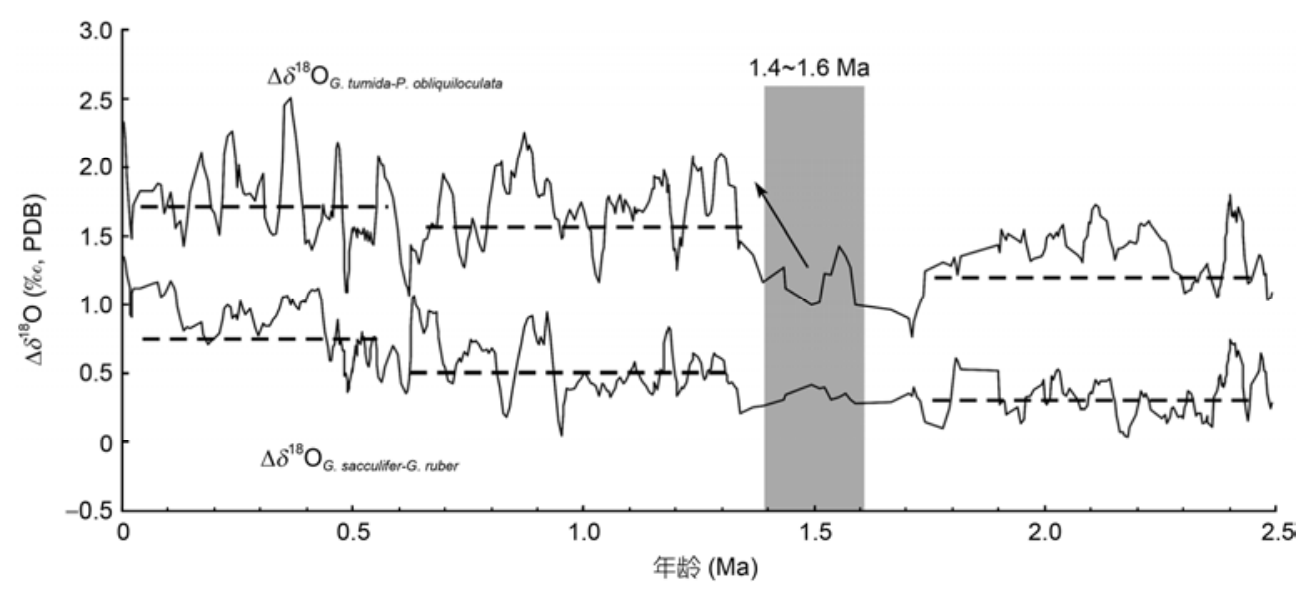

图 4 赤道东、西太平洋 ODP 851 站和 ODP 807 站之间表层水和次表层水氧同位素东-西向梯度变化

氧同位素差值曲线经过 5 点平滑处理, 灰色条带指示 1.4 1.6 Ma, 水平虚线代表不同时间段内的平均值, 箭头指示 $1.4 \mathrm{Ma}$ 左右次表层水氧同位 素差值的突然增大 
世赤道太平洋东-西向温度不对称格局首先是发生在 次表层水, 然后才导致表层海水温度的东-西向不对 称性格局逐步形成 ${ }^{[25]}$ (图 4).

现代海洋观测和数值模拟研究表明, 赤道西太 平洋的次表层水主要是南大洋高纬海区的 “模态” 水 下潜北上而形成, 然后以赤道逆流的形式进人东太 平洋、形成上升流 ${ }^{[26,27]}$. 由于南大洋是全球两大深层 水上涌区之一, 这一海区的深部环流格局改组就有 可能影响到赤道太平洋的次表层水、表层水, 改变赤 道海区上部水体的垂向结构 ${ }^{[28]}$. 因此, 早更新世 1.6 1.4 Ma 期间赤道太平洋东-西向温度不对称格局 的形成, 不仅在时间上与当时南大洋深部水的重大 改组 ${ }^{[7]}$ 相吻合, 而且有可能存在某种因果关系 ${ }^{[9]}$.

既然富 $\mathrm{Si}$ 的南极模态水可以下潜北上“渗漏”到 热带成为次表层水, 就可能引起低纬海区硅藻勃发, 改变大洋碳储库 ${ }^{[9]}$, 从而减少大气中二氧化碳含量, 这就是所谓的“硅泄露”假说 ${ }^{[29,30]}$. 实际上, 早更新世 赤道太平洋东-西向温度不对称格局, 也反映在赤道 太平洋浮游有孔虫碳同位素变化上. 如图 5 所示, 赤 道东、西太平洋的浮游有孔虫表层水种和次表层水种 碳同位素差异在 1.6 1.4 Ma 以后较之以前明显减小, 意味着热带太平洋上部水体的碳储库发生重大变化.
特别的是, 从图 5 中可以明显识别出 $2.5 \mathrm{Ma}$ 以来碳同 位素的 6 个重值期 $\delta^{13} \mathrm{C}_{\max }-\mathrm{I} \sim \delta^{13} \mathrm{C}_{\max }-\mathrm{VI}$, 并且 $1.6 \mathrm{Ma}$ 之前的碳重值期以 400 $\mathrm{ka}$ 的周期为主, $1.6 \mathrm{Ma}$ 之后 碳重值每隔 500 ka 才出现. 这种现象在包括南沙海 区的全球大洋碳储库的变化中均有出现 ${ }^{[9,31]} .1 .6 \mathrm{Ma}$ 后受北半球高纬冰盖的影响, 大洋碳储库原有的 400 ka“心跳”周期 ${ }^{[32]}$ 被打乱, 产生了所谓的“心律不 齐”现象, 可能表明地球气候系统由太阳辐射量控制 向极地冰盖控制转变 ${ }^{[9]}$.

可见, 早更新世 1.6 1.4 Ma 期间赤道太平洋上 部水体垂向结构的东、西向不对称格局的最终形成, 究其原因, 可能与南大洋深部大洋环流的改组有关, 且其变化主要发生在次表层水。一旦这种类似于现 代 ENSO 的东、西太平洋不对称格局形成, 就结束了 赤道太平洋上新世 $5 \mathrm{Ma}$ 以来长期的 El Niño 状态 ${ }^{[4 \sim 6]}$, 并通过水气交换传输到中高纬海区、影响大洋碳储库 变化, 进而在全球气候演变中发挥重要作用.

\section{5 结论}

(1) 赤道西太平洋 ODP 807 站 $2.5 \mathrm{Ma}$ 以来的浮 游有孔虫次表层水种 $(P$. obliquiloculata $)$ 与表层水种

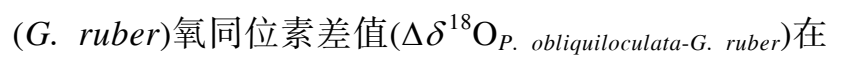

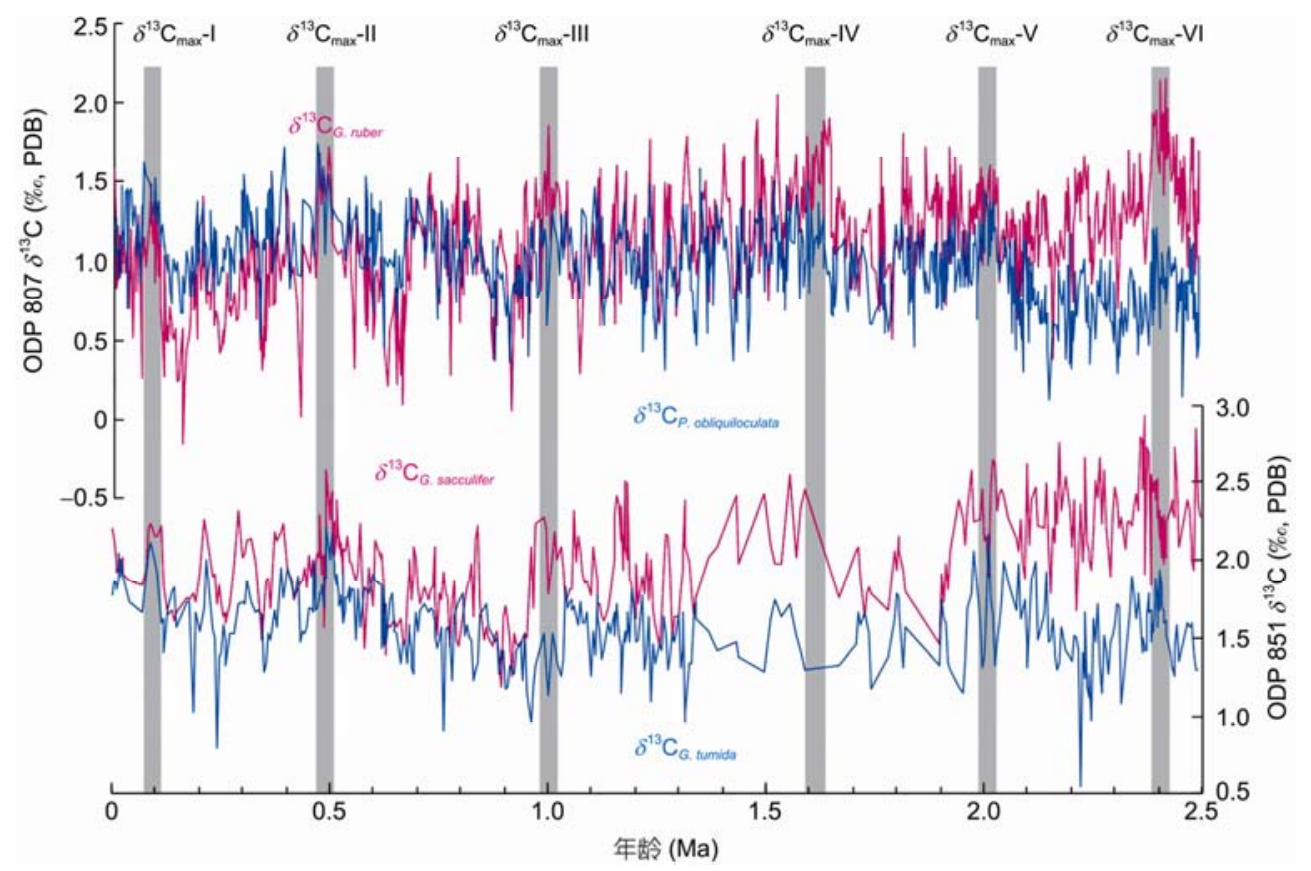

图 5 赤道太平洋 $2.5 \mathrm{Ma}$ 年以来的碳同位素比较

红色曲线为表层水碳同位素, 蓝色曲线为次表层水碳同位素, 灰色条带标示碳同位素重值期的 $\delta^{13} \mathrm{C}_{\max }-\mathrm{I} \sim \delta^{13} \mathrm{C}_{\max }-\mathrm{VI}$ 
早更新世 1.6 1.4 Ma 以来有增大的趋势. 通过与赤 道东太平洋 ODP 851 站的记录比较, 证实赤道太平 洋类似于现代的表层海水东-西向温度梯度和上部水 体结构的东、西不对称格局在 1.6 1.4 Ma 期间最终 形成. 由于次表层水的氧同位素东-西向梯度变化明 显大于表层水的, 说明早更新世赤道太平洋东-西向 温度不对称格局首先发生在次表层水, 然后才导致 表层海水温度的东-西向不对称格局逐步形成.
(2) 早更新世 1.6 1.4 Ma 以来，赤道东、西太平 洋的浮游有孔虫表层水种和次表层水种的碳同位素 差异较之前明显减小, 其偏心率长周期也从 $400 \mathrm{ka}$ 变为 500 ka, 反映了大洋碳储库的重大变化. 因而, 早更新世 1.6 1.4 Ma 期间, 赤道太平洋东-西向温度 不对称格局的形成，不仅在时间上与当时南大洋的 大洋环流改组相吻合, 而且可能存在某种因果关系, 从而改变大洋碳储库, 影响全球气候变化.

\section{参考文献}

1 Yan X H, Hou C, Zheng Q. Temperature and size variabilities of the Western Pacific Warm Pool. Science, 1992, 58: 1643-1645

2 Cane M. A role for the tropical Pacific. Science, 1998, 282: 59-61

3 Webster P, Magana V, Palmer T, et al. Monsoon: Process, predictability and the prospects for prediction. J Geophys Res, 1998, 103: $14451-14510$

4 Wara M W, Ravelo A C, Delaney M L. Permanent El Niño-Like conditions during the Pliocene Warm Period. Science, 2005, 309: 758-761

5 Lawrence K T, Liu Z, Herbert T D. Evolution of the Eastern Tropical Pacific through Plio-Pleistocene glaciation. Science, 2006, 312: 79-83

6 Ravelo A C, Andreasen D H, Lyle M, et al. Regional climate shifts caused by gradual global cooling in the Pliocene epoch. Nature, 2004, 429: 263-267

7 Hodell D A, Venz-Curtis K A. Late Neogene history of deepwater ventilation in the Southern Ocean. Geochem Geophys Geosys, 2006, 7 : Q09001, doi: 10.1029/2005GC001211

8 Fedorov A V, Dekens P S, McCarthy M, et al. The Pliocene paradox (mechanisms for a permanent El Nino). Science, 2006, 312: $1485-1489$

9 Wang P X, Tian J, Lourens L J. Obscuring of long eccentricity cyclicity in Pleistocene oceanic carbon isotope records. Earth Planet Sci Lett, 2010, 290: 319-330

10 McPhaden M J, Busalacchi A J, Cheney R, et al. The tropical ocean-global atmosphere observing system: A decade of progress. J Geophys Res, 1998, 103: 14169-14240

11 Li C, Mu M. El Niño occurrence and subsurface ocean temperature anomalies in the Pacific warm pool. Chin J Atmos Sci, 1999, 23: $513-521$

$12 \mathrm{Xu}$ J, Holbourn A, Kuhnt W, et al. Changes in the thermocline structure of the Indonesian Outflow during Terminations I and II. Earth Planet Sci Lett, 2008, 273: 152-162

13 安阳, 翦知潜. 末次冰消期南海南部的普林虫低值事件. 科学通报, 2009, 17: 2527-2532

14 Ravelo A C, Shackleton N J. Evidence for surface-water circulation changes at Site 851 in the eastern tropical Pacific ocean. Proc Ocean Drill Prog, Sci Result, 1995, 138: 503-514

15 Prentice M L, Friez J K, Simonds G G, et al. Neogene trends in planktonic foraminifer $\delta^{18} \mathrm{O}$ from site 807: Implications for global ice volume and western equatorial Pacific sea surface temperatures. Proc Ocean Drill Prog, Sci Result, 1993, 130: 281-283, 295-298

16 Lisiecki L E, Raymo M E. A Pliocene-Pleistocene stack of 57 globally distributed benthic $\delta^{18}$ O records. Paleoceanography, 2005, 20: PA1003, doi:10.1029/2004PA001071

17 Bé A W H. An Ecological, Zoogeographic and Taxonomic Review of Recent Planktonic Foraminifera. London: Academic Press, 1977. 1-100

18 Cléroux C, Cortijo E, Duplessy J C, et al. Deep-dwelling foraminifera as thermocline temperature recorders. Geochem Geophys Geosys, 2007, 8, doi: 10.1029/2006GC001474

19 Moore T C Jr, Pisias N G, Keigwin L D Jr. Cenozoic Variability of Oxygen Isotopes in Benthic Foraminifera. Washington D C: Ntional Academy Press, 1982. 172-182

20 Locarnini R A, Mishonov A V, Antonov J I, et al. World Ocean Atlas 2005, Temperature. In: Levitus S, ed. NOAA Atlas NESDIS 61 Washington D C: U S Government Printing Office, 2006. 1-182 
21 Berger W H, Bickert T, Jansen E, et al. The central mystery of the Quaternary Ice Age. Oceanus, 1993, 36: 53-56

22 Rutherford S, Hondt S D. Early onset and tropical forcing of 100000-year Pleistocene glacial cycles. Nature, 2000, 408: 72-75

23 Jian Z M, Wang P X, Saito Y, et al. Holocene variability of the Kuroshio Current in the Okinawa Trough, northwestern Pacific Ocean. Earth Planet Sci Lett, 2000, 184: 305-319

24 Tian J, Wang P X, Chen R, et al. Quaternary upper ocean thermal gradient variations in the South China Sea: Implications for east Asian monsoon climate. Paleoceanography, 2005, 20: PA4007, doi: 10.1029/2004 PA001115

25 Medina-Elizalde M, Lea D W, Fantle M S. Implications of seawater Mg/Ca variability for Plio-Pleistocene tropical climate recostruction. Earth Planet Sci Lett, 2008, 269: 585-595

26 Gu D, Philander S G H. Interdecadal climate fluctuations that depend on exchanges between the tropics and the extratropics. Science, 1997, 275: 805-807

27 Pedlosky J. A History of Thermocline Theory. New York: Springer, 2006. 139-152

28 Sarmiento J L, Gruber N, Brzezinski M A, et al. High-latitude controls of thermocline nutrients and low latitude biological productivity. Nature, 2003, 427: 56-60

29 Anderson R F, Chase Z, Fleischer M Q, et al. The Southern Ocean's biological pump during the Last Glacial Maximum. Deep Sea Research, Part II, 2002, 49: 1909-1938

30 Crosta X, Beucher C, Pahnke K, et al. Silicic acid leakage from the Southern Ocean: Opposing effects of nutrient uptake and oceanic circulation. Geophys Res Lett, 2007, 34: L13601, doi: 10.1029/2006GL029083

31 Wang P X, Tian J, Cheng X R, et al. Major Pleistocene stages in a carbon perspective: The South China Sea record and its global comparison. Paleoceanography, 2004, 19, doi: 10.1029/2003PA000991

32 Pälike H, Norris R D, Herrle J O, et al. The heartbeat of the Oligocene climate system. Science, 2006, 314: 1894-1898 